\title{
Una nueva mirada a la competencia transversal de trabajo en equipo desde los enfoques de participación y cuidados: El caso del Máster en Cooperación al Desarrollo por la UPV
}

\author{
Carola Calabuig Tormo a , Sergio Belda Miquel ${ }^{\mathrm{b}}$ y Álvaro Fernández-Baldor \\ $\mathrm{a}, \mathrm{b}, \mathrm{c}$ Departamento Proyectos de Ingeniería, Universitat Politècnica de València
}

\begin{abstract}
This communication presents the work carried out to improve and evaluate the development of the transversal competence "teamwork" in the students of the Master's Degree in Development Cooperation by the Universitat Politècnica de València. During the academic years 2015-2016 and 2016-2017 it has been realized emphasizing the approaches of participation and care ethics. The results are being satisfactory and encouraging, due in part to the collaboration with an external Development NGO. But some improvements can be introduced such as deepening the approaches adopted and their transfer to the competence, to extend the proposal to the whole master progressively or to improve the monitoring of the process and the accompaniment of the students.
\end{abstract}

Keywords: teamwork, transversal competences, participation, care ethics, evaluation

\begin{abstract}
Resumen
La comunicación presenta el trabajo realizado durante los cursos académicos 2015-2016 y 2016-2017 para mejorar y evaluar el desarrollo de la competencia transversal "trabajo en equipo" en el alumnado del Máster en Cooperación al Desarrollo por la Universitat Politècnica de València, desde los enfoques de la participación y los cuidados. Los resultados están siendo satisfactorios y alentadores, debido en parte a la colaboración con una ONGD externa; si bien evidencian elementos susceptibles de reflexión y mejora, como son profundizar en los enfoques adoptados y su traslado a la competencia estudiada, hacer extensible el trabajo en esta competencia a todo el máster progresivamente o mejorar el seguimiento y el acompañamiento al alumnado.
\end{abstract}

Palabras clave: trabajo en equipo, competencias transversales, participación, enfoque de cuidados, evaluación

\section{Introducción}

En el marco de los estudios de gestión de la cooperación al desarrollo existe un intenso debate sobre el rol que la formación superior debe tener en la formación de profesionales del desarrollo. Esta cuestión conecta a su vez con las reflexiones acerca de qué capacidades son 
necesarias para la gestión del desarrollo, y cómo pueden desarrollarse en un proceso de educación formal (Clarke y Oswald, 2010).

En este debate existe una corriente hegemónica, dominada por una perspectiva instrumental y poco crítica con la gestión del desarrollo (Gulrajani, 2010; Mosse, 2005). Según esta visión, las personas dedicadas a la gestión del desarrollo deben ser "buenos técnicos", capaces de manejar los instrumentos de gestión adecuados para identificar las mejores soluciones y guiar adecuadamente el cambio en la dirección deseada (Johnson y Thomas, 2007). Como Peris et al. (2013) mencionan, esta perspectiva implicaría que los procesos formativos en gestión de proyectos de desarrollo se centren en proveer a las y los estudiantes capacidades para manejar herramientas para alcanzar de manera eficiente objetivos prefijados de desarrollo mediante una secuencia lineal de pasos, o al menos mediante una planificación estricta (Clarke and Oswald, 2010).

En cambio, otra corriente más crítica consideraría que estas perspectivas, tecnocráticas y gerenciales, no contribuirían a abordar las causas estructurales de la pobreza y la exclusión sino que, al contrario, reforzarían relaciones desiguales de poder. Esta perspectiva considera que los gestores del desarrollo deberían poner en el centro la naturaleza siempre compleja y política de los procesos de desarrollo, en los que las relaciones de poder juegan un lugar fundamental (McCourt y Gulrajani, 2010; Eyben et al, 2015). Esta visión entendería que los programas de educación superior en gestión del desarrollo, en lugar de centrarse únicamente en el desarrollo de habilidades para el manejo de instrumentos gerenciales de gestión, deberían focalizarse más bien en generar competencias para entender, desvelar y moverse en contextos complejos y embebidos en relaciones de poder, desde una perspectiva, abierta, flexible, colaborativa y adaptativa. Entre estas competencias, tiene un rol fundamental el trabajo en equipo y la actitud cooperativa para un trabajo productivo, respetuoso y transformador.

Esta perspectiva apuntaría por lo tanto a replantear las metodologías de enseñanzaaprendizaje actualmente empleadas en la gestión del desarrollo, así como las metodologías dominantes en el ámbito universitario en general. Distintos autores del ámbito del desarrollo han apuntado que existen numerosos métodos y aproximaciones pedagógicas para desarrollar capacidades que vayan más allá del gerencialismo en la práctica del desarrollo (ver ejemplos específicos en Ortiz Aragon, 2010; Pearson, 2010; Pettit, 2010; Soal, 2010), y que van desde el aprendizaje experiencial a métodos creativos que apelan a las emociones y el uso del cuerpo.

\subsection{Propuesta educativa del Máster en Cooperación al Desarrollo por la UPV}

Es en esta perspectiva crítica del desarrollo y la formación para la gestión del desarrollo que se sitúa la propuesta educativa del Máster Universitario en Cooperación al Desarrollo por la Universitat Politècnica de València (UPV), Especialidad en Gestión de Proyectos y Procesos de Desarrollo (en adelante MCAD). El MCAD es un título oficial de 90 ECTS, orientado a formar profesionales de la gestión del desarrollo con competencias que les permitan una práctica crítica de la cooperación. Aunque arranca en el curso 2011-2012 como título oficial, 
desde 2007 ya se impartía un máster título propio con contenidos y enfoque muy similares, que fue el precedente del actual título.

El MCAD se imparte en dos cursos académicos. Durante el primer año se cursan presencialmente 60 ECTS de asignaturas en la UPV: en el primer cuatrimestre, Conceptos básicos del desarrollo (12 ECTS) y Cooperación al desarrollo (12 ECTS). En el segundo semestre, las asignaturas de la especialidad: Procesos de desarrollo (12 ECTS), Gestión del ciclo del proyecto de cooperación al desarrollo (14 ECTS) y Metodologías de investigación en proyectos de desarrollo (10 ECTS). En el segundo año se cursan de manera no presencial 30 ECTS, divididos entre prácticas externas realizadas en una organización de desarrollo, tanto en el extranjero como en España, y el Trabajo Fina de Máster.

El MCAD se desarrolla en el entorno de la UPV, una universidad técnica con una perspectiva aún muy instrumental, racionalista y limitada de las capacidades que una universidad debe desarrollar en su alumnado; muy orientada a la empleabilidad, y con un uso aún muy extendido de prácticas de enseñanza-aprendizaje poco activas. Sin embargo, la UPV ha ido generando también estrategias y espacios alternativos, para tratar de que su profesorado genere y proponga una perspectiva más amplia de la enseñanza superior y de los procesos de enseñanza-aprendizaje. Por ejemplo, está tratando de impulsar progresivamente el trabajo en trece "competencias transversales" en todas las titulaciones y asignaturas, así como en el uso de metodologías activas de enseñanza-aprendizaje para desarrollarlas.

El MCAD ha tratado de alinearse con estos procesos facilitados por la UPV y se ha sumado al trabajo en determinadas competencias transversales. Entre estas, el MCAD ha considerado clave la denominada CT06 Trabajo en equipo y liderazgo, ya que está especialmente alineada con su visión del desarrollo, la gestión del desarrollo y la enseñanza-aprendizaje. Como veremos, el máster ha tratado de desarrollar la competencia en su alumnado mediante distintos métodos, basados en dos enfoques clave que reorientan el sentido habitualmente dado a esta competencia: desde un enfoque eficientista, a un enfoque participativo y de cuidados.

Así, en este contexto, el presente trabajo expone los resultados obtenidos al poner en práctica entre los años 2016 y 2017 la competencia transversal denominada Trabajo en equipo y liderazgo, desde un enfoque de participación y cuidados, en el seno del Máster Universitario en Cooperación al Desarrollo por la UPV.

\subsection{El Equipo de Innovación y Calidad Educativa del MCAD}

Para profundizar en el trabajo en esta competencia en el profesorado del máster, se decide coordinar esfuerzos en el marco del Equipo de Innovación y Calidad Educativa denominado "Innovación pedagógica en el Máster en Cooperación al Desarrollo: integrando la participación y la acción en el proceso de enseñanza-aprendizaje" (en adelante EICEMACODE), creado en junio de 2012. Estos equipos de innovación educativa se han ido desarrollando en la UPV por iniciativa del Vicerrectorado de Estudios, Calidad y Acreditación, con el respaldo del Instituto de Ciencias de la Educación. 
El EICE-MACODE, desde sus inicios, volcó su trabajo en apoyar el enfoque participativo y crítico propio del Máster que se ha señalado. Integrado desde sus inicios por profesorado del MCAD perteneciente a diversos departamentos y categorías docentes, el EICE-MACODE ha ido aportando al máster un acervo de procesos y propuestas concretas en sus diversas asignaturas y en el planteamiento mismo del máster. Los resultados de este trabajo se pueden ver en distintas publicaciones (por ejemplo, Belda et al., 2012; Peris et al.; 2013; Pellicer y Calabuig, 2015; Boni-Aristizábal, Calabuig-Tormo y Pellicer-Sifres 2017; Belda et al., 2016), y han contribuido a la mejora progresiva del Máster, por ejemplo, incorporando nuevas metodologías de enseñanza-aprendizaje, así como la investigación-acción participativa.

El equipo de innovación se reorganiza durante el curso 2015-2016, actualiza sus miembros (en la actualidad lo componen siete docentes del MCAD) y decide concentrar sus esfuerzos, tras las recomendaciones del ICE de la UPV, en profundizar e innovar en competencias transversales de la propuesta UPV que son especialmente claves en el enfoque del Máster. Es por ello que se decide apostar, de manera progresiva y con diferentes grados de intensidad, por las competencias: Trabajo en equipo y liderazgo; Responsabilidad ética, medioambiental y profesional; Pensamiento crítico y Conocimiento de problemas contemporáneos. En un primer momento, se decide centrar los esfuerzos de manera más específica en trabajar la competencia de trabajo en equipo y liderazgo, y hacerlo en tres asignaturas: Cooperación al desarrollo, Procesos de desarrollo y Gestión del ciclo del proyecto de cooperación al desarrollo. Los autores de la presente comunicación son los profesores responsables de cada una de ellas y miembros a su vez del EICE-MACODE. Se decide además trabajar el desarrollo de la competencia en el alumnado desde dos enfoques específicos: el enfoque participativo y el enfoque de cuidados, combinando la experiencia y esfuerzo del personal docente del Máster con el apoyo de profesionales externas.

\section{Objetivos}

La presente comunicación recoge el trabajo realizado durante los cursos académico 20152016 y 2016-2017 (hasta marzo de 2017 inclusive) para mejorar y evaluar el desarrollo en el alumnado del MCAD de la competencia transversal trabajo en equipo y liderazgo, en tres asignaturas del primer curso del MCAD, desde los enfoques de la participación y los cuidados, siendo los objetivos al poner en marcha la iniciativa los siguientes.

- Incorporar la competencia de trabajo en equipo (colaborativo) y liderazgo (social), de forma más intensa en la formación del máster.

- Integrar en la competencia trabajo en equipo y liderazgo los enfoques de participación y de cuidados.

- Disponer de herramientas para la adecuada evaluación cualitativa y/o cuantitativa de dicha competencia, tanto en el seno del máster como a futuro.

- Potenciar entre el alumnado la reflexión sobre el proceso seguido en el trabajo en equipo, tanto el llevado a cabo de forma presencial en el aula, como fuera de ella.

(cc) EY-NC-ND 2017, Universitat Politècnica de València 
- Identificar durante la iniciativa elementos de mejora para contribuir a un mejor desempeño en la puesta en práctica y evaluación de la competencia de trabajo en equipo y liderazgo.

\section{Desarrollo de la innovación}

\subsection{El enfoque de la innovación: participación y cuidados}

Para el desarrollo de la competencia de trabajo en equipo en el alumnado se ha construido tanto sobre la experiencia, enfoques y perspectivas que venían siendo propias del máster como sobre otras que han despertado la atención del mismo. En concreto, se trató de trabajar sobre los mencionados enfoques de participación y de cuidados, de trayectoria más incipiente. Es necesario, no obstante, hacer ver que desde el EICE-MACODE se ha priorizado la dimensión trabajo en equipo frente a liderazgo, aunque esta segunda ha estado presente en todo momento. Ello obedece a querer evitar dispersión y por la limitación de recursos para abordar ambos elementos con la debida especificidad.

En primer lugar, se trabajó desde el enfoque de participación, que ha sido esencial en la definición del enfoque pedagógico general y en la metodología de enseñanza-aprendizaje del MCAD (ver Peris et al., 2013; Belda et al., 2016). Por un lado, en el máster se aprenden y aplican un buen número de metodologías y técnicas participativas: por ejemplo, se aprenden y aplican, en clase y en contextos reales, herramientas de planificación participativa como los mapeos comunitarios, los sociogramas o los flujogramas; o se desarrollan proyectos de investigación-acción participativa. Por otro lado, se emplean técnicas participativas como métodos de enseñanza-aprendizaje, tales como debates o el teatro social. Así, tanto la enseñanza de técnicas de participación como el uso mismo de la participación para la enseñanza-aprendizaje han tenido su reflejo en el trabajo en equipo como competencia: no solo se diseñan y aplican en grupo herramientas participativas, sino que se emplea el trabajo en equipo como espacio básico para generar participación.

En segundo lugar, se ha trabajado desde el denominado enfoque de cuidados, que algunas ONGD están implementando desde la llamada pedagogía de cuidados. Se trata de un planteamiento sobre el que había un creciente interés, pero no había sido trabajado hasta la fecha como estrategia pedagógica por el profesorado del máster. Por ello, se solicitó el apoyo de InteRed, ONG española de referencia en materia de educación para el desarrollo y educación para la ciudadanía global, que cuenta con delegación en Valencia, para trabajar la competencia desde esta perspectiva. Este enfoque pone en el centro una combinación de la ética de la justicia y la ética del cuidado, centrándose en el reconocimiento del derecho individual y colectivo al cuidado (Carrasco, Borderías y Torns, 2011), la dimensión política de lo personal y la dimensión ecológica. Esto implica poner en el centro la igualdad dentro de las relaciones personales, desmontando jerarquías y privilegios, así como la atención de las necesidades que todas las personas pueden tener. El reconocimiento del otro/a como sujeto de derecho al cuidado lleva además a atender a la singularidad de cada persona o comunidad, a tener que dar espacio a la diversidad para que la diferencia no se convierta en desigualdad (de Blas, 2016). En general, un enfoque de cuidados supone implicarse en el 
bienestar de las demás personas, desde posturas ecofeministas (Herrero, 2013), siendo consciente que el bienestar es una experiencia personal, vinculada a los intereses y deseos personales, a la razón, al cuerpo y a las emociones.

\subsubsection{Proceso preparatorio y de ensayo durante el curso 2015-2016}

Durante el curso 2015-2016 el trabajo llevado a cabo en el seno del EICE-MACODE, y trasladado al resto de profesorado y al alumnado del máster, combinó la reactivación de la planificación del equipo, como ya fue expuesto, con la aplicación de la evaluación de las dimensiones trabajo en equipo y participación, mediante rúbricas definidas al efecto para la asignatura Procesos de Desarrollo (cuatrimestre B). Dentro de la mencionada asignatura (aunque también se llevó a cabo trabajo en equipo en otras), fueron dos los espacios en los que se aplicó especialmente la participación y evaluación de la misma: Por un lado, la unidad temática 2 relativa a procesos de planificación, en la que el alumnado realizaba trabajos en el aula por equipos emulando talleres de planificación estratégica participativa. La particularidad en el curso 2015-2016 es que debían hacerlo en un barrio de la ciudad de Valencia, previa visita por su cuenta (por equipos) al mismo, lo que confería un carácter de mayor realismo. En las sesiones se intercambiaban los roles: en algunos momentos todos eran parte del equipo de planificación; en otros, una persona ejercía de facilitadora para aplicar una herramienta de diagnóstico o planificación, mientras que el resto de miembros del equipo actuaban con vecinas del barrio. Al final de las sesiones, se dedicaba algún tiempo para valorar y reflexionar cómo se habían sentido en el rol. Los grupos se autoevaluaron también en dos sesiones empleando métodos visuales. Por otro lado, la unidad temática 3 denominada Investigación-Acción Participativa (IAP), sobre la cual se venía ya trabajando durante los dos cursos académicos anteriores. En este módulo el alumnado se dividía en equipos de 4-5 personas, escogía una entidad del entorno de Valencia de las ofertadas (principalmente ONGD u organizaciones sociales) y durante dos meses realizaba un trabajo real práctico en diálogo con la entidad de acogida; es decir, los objetivos de la investigación de cada equipo de estudiantes se definían conjuntamente con la entidad y la investigación se llevaba a cabo desde un diseño flexible y emergente.

En esta asignatura la participación ha sido una dimensión que se lleva evaluando (con una contribución de un $10 \%$ a la nota final) desde que el máster es oficial, tanto la individual en el aula, como la desarrollada en el marco de los trabajos en equipo; sin embargo, es en el curso 2015-2016 que se decide revisar las rúbricas establecidas y profundizar en cómo evaluar la participación individual de cada estudiante. Para el curso 2015-2016 se redefinió el cuestionario de evaluación con sus rúbricas, basándose en parte en materiales facilitados por el ICE (entre ellos, Ábalos et al, 2015), que quedó compuesto por ocho dimensiones: gestión, ideas propias, voz, aceptación de otras ideas, crítica constructiva, actitud, cooperación y resolución de problemas. Este cuestionario ha sido la base, con sus modificaciones, para la etapa en la que actualmente se encuentra la innovación objeto de estudio.

(c) EY-NC-ND 2017, Universitat Politècnica de València 


\subsubsection{Actividades propuestas para el curso 2016-2017}

Fruto de la evaluación de final y de los aprendizajes obtenidos, que serán expuestos en el siguiente apartado, se decide a final del curso 2015-2016 planificar desde el EICE-MACODE el curso siguiente, con una apuesta decidida por la competencia CT06 Trabajo en equipo y liderazgo. Si bien las diversas asignaturas del máster seguirían siendo punto de control de otras competencias, se toma la decisión de profundizar en la mencionada anteriormente: garantizando una mejor coordinación entre el profesorado; buscando una mayor coherencia entre métodos empleados en las diferentes asignaturas; incorporando las demandas del alumnado fruto de co-evaluaciones realizadas en los comités de seguimiento de las asignaturas (por ejemplo: precisar las rúbricas, dar más herramientas para el trabajo en equipo o dimensionar mejor el trabajo en equipo autónomo); revisando el sistema de rúbricas para la evaluación; e implicando a las ONG de Desarrollo que forman parte del Consejo Asesor del máster.

Es por ello que para el curso 2016-2017 se han redefinido unidades temáticas de algunas asignaturas y se ha realizado el esfuerzo de revisar internamente tanto los métodos docentes como de evaluación, de forma que el trabajo en equipo fuera puesto en práctica con el rigor y profundidad requeridos, así como de forma coherente con los planteamientos del máster expuestos con anterioridad.

Las actividades previstas para el curso 2016-2017, algunas ya finalizadas y otras en estado de implementación, son las siguientes:

Taller sobre trabajo en equipo, específicamente diseñado para el alumnado de primer año del máster e impartido por personal especializado de la ONGD Intered. El taller, llevado a cabo el 14 de octubre de 2016 y con 8 horas de duración, tuvo lugar en la cuarta semana de clase, de manera que servía tanto para abordar los conceptos considerados básicos, como para facilitar la integración y conocimiento mutuo de las y los estudiantes recién llegados. El taller se diseñó desde un enfoque de trabajo en equipo de corte colaborativo y con elevada componente emocional. De forma coherente con el trabajo de esta ONGD, se decide incorporar en la formación solicitada el enfoque de cuidados.

Redefinición del sistema de evaluación de la competencia CT6 Trabajo en equipo y liderazgo, incorporando más espacios y actividades secuenciadas y reduciendo el número de ítems o dimensiones a evaluar en el cuestionario, para hacerlo menos complejo. La figura 1 recoge el esquema de trabajo planteado para las tres asignaturas que han sido elegidas como punto de control de esta competencia.

Respecto a los cuestionarios de autoevaluación y evaluación por pares, se decide reducir a cuatro los criterios de evaluación, gran parte de ellos definidos como agregado de varios ítems del cuestionario de evaluación empleado en el curso anterior. Este cuestionario es propuesto por el profesorado y revisado por la ONGD Intered, recibiendo aportes para matizar el primer criterio e incorporar el enfoque de cuidados. Se decide para 2016-2017 no emplear un sistema de rúbricas como tal, sino aplicar una escala cualitativa de valoración para el cuatrimestre A, y una escala cuantitativa para el cuatrimestre B. Ello es debido a que en las asignaturas del segundo semestre la participación sí es calificada y contribuye a la nota 
final de las mismas. La figura 2 muestra la tabla empleada para la valoración. De la misma se generó el cuestionario de autoevaluación y evaluación por pares, de forma que cada estudiante valorase el desempeño del resto de miembros de su equipo y así mismo/a (cuatro columnas) según los cuatro criterios definidos. Junto a él se incorporaron una serie de preguntas que permitieran valorar aspectos de contexto relativos al proceso que cada equipo y persona han experimentado. Este cuestionario se aplica a las tres asignaturas mencionadas, con algunas particularidades.

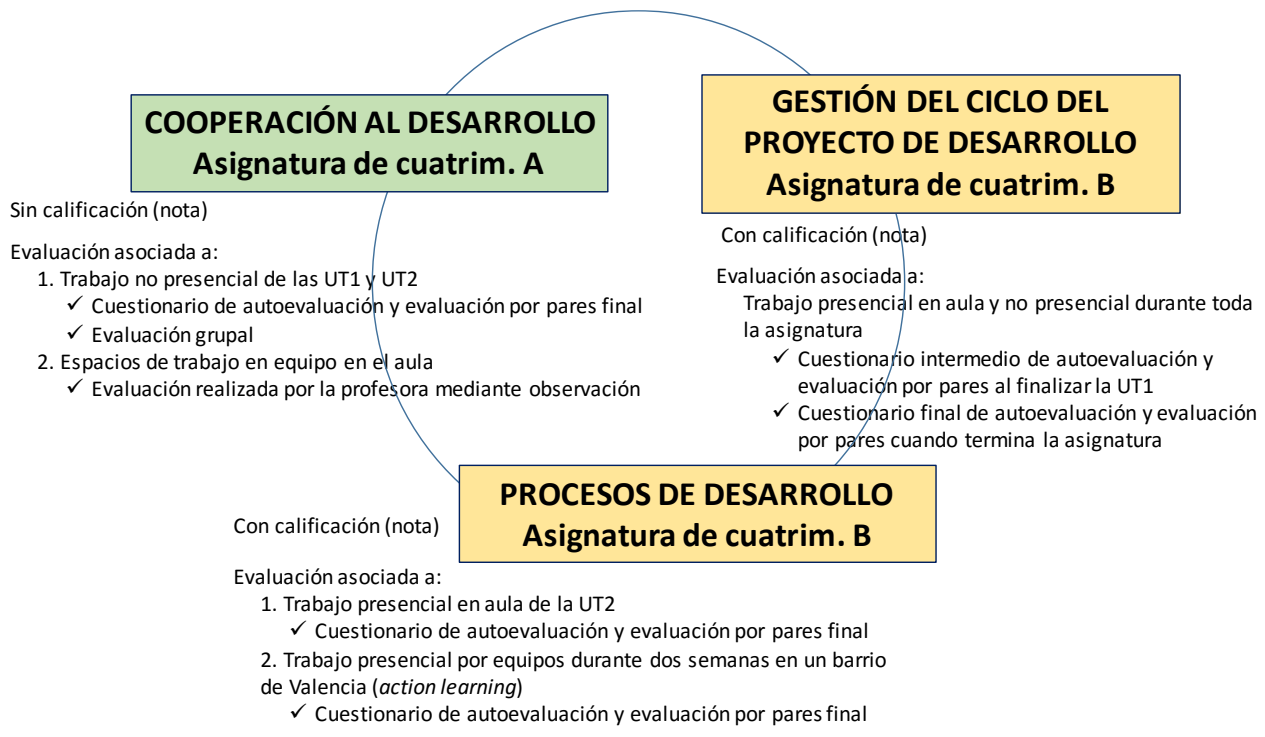

Figura 1: Planteamiento de la evaluación de la competencia en las diversas asignaturas Fuente: elaboración propia

Evaluación de los mecanismos puestos en marcha al final del cuatrimestre A, tanto en el seno del EICE-MACODE, como con el alumnado del máster (en aula y a través de los Comités de Seguimiento de asignaturas), para reformular si fuera el caso los cuestionarios de evaluación. En el caso de las asignaturas del semestre B, es el propio alumnado quien replantea el cuestionario para que no se valorase (y calificase) a personas en concreto, sino que cada persona evaluase el desempeño suyo y del equipo. Manteniendo los cuatro criterios del trabajo en equipo, esto se traduce en plantear solamente las columnas de autoevaluación y evaluación global del desempeño del equipo.

Rediseño de la propuesta de Aprendizaje-Servicio en la asignatura de Gestión del ciclo del proyecto y diseño de la propuesta de action learning o aprendizaje en la acción, vinculada a la UT3 de la asignatura Procesos, tomando en consideración los aprendizajes respecto a la competencia trabajo en equipo obtenidos en el transcurso del curso académico. No será objeto de la presente comunicación describir estas propuestas de innovación docente, a desarrollar de manera intensiva con ONG externas (caso del APS) o conviviendo con organizaciones sociales durante dos semanas de mayo-junio de 2017 en el barrio de Benicalap de Valencia.

(cc) EY-NC-ND 2017, Universitat Politècnica de València 
Diseño de un segundo taller sobre trabajo en equipo, a llevar a cabo el 25 de mayo de 2017 en la UPV como continuación del realizado en octubre. En este caso, vinculado a cómo abordar desde el enfoque de cuidados los conflictos en el seno de los equipos de trabajo

\begin{tabular}{|c|c|c|c|c|c|}
\hline \multicolumn{6}{|c|}{ Valoración de la competencia trabajo en equipo y liderazgo } \\
\hline \multirow[t]{2}{*}{ Criterio } & \multirow[t]{2}{*}{ Qué comprende el criterio } & \multicolumn{4}{|c|}{ Descriptores } \\
\hline & & A & B & $\mathrm{C}$ & $\mathrm{D}$ \\
\hline $\begin{array}{l}\text { Escucha activa, } \\
\text { empatía, cuidados } \\
\text { y respeto }\end{array}$ & $\begin{array}{l}\text { En los espacios de trabajo se muestra una persona con interés. } \\
\text { Focaliza la atención en la dinámica de grupo, sin desviarse a } \\
\text { otros asuntos. Diferencia lo que son espacios de comunicación } \\
\text { relativos al trabajo del máster de los espacios privados. Da su } \\
\text { opinión sin monopolizar las conversaciones. El lenguaje } \\
\text { empleado en las intervenciones en general es inclusivo y } \\
\text { respetuoso. Es capaz de empatizar con l@s compañer@s y } \\
\text { actúa desde la cultura de los cuidados, no buscando solamente } \\
\text { un enfoque "eficientista" de la tarea. }\end{array}$ & & & & \\
\hline $\begin{array}{l}\text { Gestión de } \\
\text { conflictos }\end{array}$ & $\begin{array}{l}\text { Tiene en cuenta diferencias culturales o de otro tipo que } \\
\text { pueden dificultar un adecuado entendimiento y provocar } \\
\text { malestar. Busca integrar a todas las personas que forman parte } \\
\text { del equipo, incorporándolas a la conversación o a la sesión } \\
\text { concreta. Es capaz de aclarar su punto de vista de manera } \\
\text { adecuada frente a sus compañeras, así como reaccionar bien } \\
\text { ante visiones contrapuestas. Es capaz de aportar soluciones a } \\
\text { posibles conflictos generados entre los miembros del equipo }\end{array}$ & & & & \\
\hline Liderazgo social & $\begin{array}{l}\text { Sus intervenciones invitan a otros/as a profundizar en } \\
\text { cuestiones particulares. Valora, recoge e integra ideas } \\
\text { formuladas por sus compañeros/as para profundizar en ellas. } \\
\text { Motiva a las compañeras, promueve la distribución de roles y } \\
\text { la cohesión del grupo. }\end{array}$ & & & & \\
\hline $\begin{array}{l}\text { Responsabilidad, } \\
\text { compromiso y } \\
\text { rendición de } \\
\text { cuentas }\end{array}$ & $\begin{array}{l}\text { Se muestra como una persona proactiva en el trabajo en equipo } \\
\text { y comprometida con los objetivos a conseguir. Es responsable } \\
\text { con las tareas encomendadas y da cuentas de las mismas, } \\
\text { comunicando avances, resultados, etc., al resto del equipo. Es } \\
\text { puntual para el inicio de las reuniones. }\end{array}$ & & & & \\
\hline $\begin{array}{l}\text { Descriptores cualit } \\
\text { A: Ocurre casi siem } \\
\text { B: Ocurre con frecu } \\
\text { C: Ocurre con poca } \\
\text { D: No ocurre casi nu }\end{array}$ & $\begin{array}{l}\text { tivos: } \\
\text { re / Se da de forma ejemplar } \\
\text { encia / Se da de forma adecuada } \\
\text { recuencia/ Se da de forma mejorable } \\
\text { nca/ Se da de forma claramente insatisfactoria }\end{array}$ & & & & \\
\hline
\end{tabular}

Figura 2: Tabla tipo para la valoración de la competencia trabajo en equipo Fuente: elaboración propia

\section{Resultados}

Expuestas las actividades previstas y/o ya desarrolladas, se ofrecen a continuación los primeros resultados obtenidos al aplicar la competencia de trabajo en equipo y liderazgo en el máster desde la participación y el enfoque de cuidados, mostrando solo aquellos más relevantes. Los aprendizajes obtenidos durante 2015-2016 han sido considerados como insumos para redefinir y configurar las acciones previstas para 2016-2017, por lo que en el presente apartado los resultados se centrarán en lo evidenciado en el actual curso académico. 


\subsection{Resultados relativos al desempeño de la competencia}

$\mathrm{Si}$ bien las competencias se adquieren (caso de lograrse, y especialmente con las transversales) con la contribución del conjunto de materias de una titulación, consideramos que es posible evidenciar algunos resultados de aprendizaje obtenidos con la experiencia llevada a cabo en dos de las asignaturas, si comparamos éstos con lo acontecido en cursos académicos anteriores.

\subsubsection{Elementos positivos identificados}

Si atendemos a la calidad académica de los trabajos como producto tangible (la memoria entregada), estos han sido muy similares a años anteriores, pero con la particularidad de que sí se ha logrado un mayor trabajo en equipo colaborativo. Es decir, los equipos no han llevado a cabo un reparto sin más de tareas, sino que se han reunido fuera del aula, han dialogado y han afrontado los conflictos cuando éstos han surgido, ya fuera durante la realización del trabajo o al final del mismo. No ha habido en ningún caso personalismos, sino que se han superado los obstáculos anteponiendo la consideración del otro/a frente a una crítica fácil ante las diferencias de pareceres. Las causas de que el posible conflicto no haya quebrado la confianza de los miembros o haya afectado gravemente a la dinámica del equipo, cuando se ha producido algún problema, no se han identificado de forma fehaciente. Sin embargo, se aprecia que el taller sobre trabajo en equipo desarrollado en octubre de 2016 y los espacios de encuentro del alumnado, han generado dinámicas muy positivas de convivencia que han favorecido ir más allá del conflicto concreto y abordarlo.

"Creo que el taller sobre trabajo en equipo ha sido positivo para la dinámica de relación de grupo en el máster y a que exista una buena disposición general. Gracias al taller, entiendo que cada compañera se ha mostrado tal como es, una prolongación del estar de cada una en el máster, o así es como es para mí". (testimonio de estudiante 1, asign Cooperación curso 2016-2017)

Cada estudiante y equipo de trabajo identificó numerosos elementos positivos como aprendizaje de la experiencia de trabajo en equipo, tanto mediante los cuestionarios como en la sesión final de evaluación llevada a cabo el 1 de diciembre de 2016. Se destaca la autocrítica y honestidad de las aportaciones del estudiantado, así como buenas reflexiones. De entre todo lo identificado, han sido capaces de discriminar aquellos elementos más relacionados con los contenidos del taller llevado a cabo en octubre de 2016, que como se expuso en anteriores apartados, incorporó el enfoque de cuidados a la formación sobre trabajo en equipo. A continuación se muestra un listado de elementos identificados por todos los equipos durante la dinámica de trabajo en equipo en la asignatura del primer cuatrimestre. Las palabras subrayadas corresponden a aquellos valores y actitudes relacionados con el taller de trabajo en equipo realizado, encontrándose numerosas coincidencias entre los equipos al respecto: participación colaborativa, complicidad, empatía, buen clima y dinámica de trabajo, optimismo al afrontar la tarea, sinceridad (con tacto), inclusión, aprendizaje mutuo, escucha $\underline{\text { activa, }}$ espacios externos buscando la convivencia, todas han trabajado, resiliencia, iniciativa, flexibilidad en las agendas, compañerismo, cohesión (a pesar de las dificultades), respeto,

(cc) EY-NC-ND 2017, Universitat Politècnica de València 
confianza mutua, enriquecimiento mutuo, compromiso, adecuada división del trabajo, sentido crítico, conocimiento mutuo, apoyo mutuo, esfuerzo, resolución de problemas, ser activos y propositivos, dinamismo, buena predisposición, ser capaces de pedir ayuda, relación y comunicación, cuidar el lenguaje, paciencia, considerar las circunstancias de cada persona que han podido afectar a su ritmo, entendimiento, comprensión, mostrarse cada una $\underline{\text { como es, }}$ cumplimiento de tareas, responsabilidad, conexión entre compañeras, $\underline{\text { hablar de los }}$ sentimientos (educación emocional), solidaridad y relaciones de horizontalidad, enriquecimiento de ideas, asertividad, predisposición al debate, agilidad.

De entre todos los elementos, ha sido una constante la constatación de que ha habido un mayor diálogo y escucha activa en el seno de los equipos de trabajo. Esta actitud favorece la comprensión de las situaciones de las otras personas, la empatía con las mismas (claramente en sintonía con el enfoque de cuidados) y el aumento de confianza personal y en el grupo, derivando en un mejor clima de trabajo y consecución de metas establecidas:

"Nuestro equipo ha trabajado de una forma muy unida y respetuosa; no hemos tenido en ningún momento problemas y hemos sabido resolver los momentos de enfrentamiento sin caer en conflictos; la clave fue escuchar para entender la posición recíproca". (testimonio de estudiante 2, asign Cooperación curso 2016-2017)

"Creo que lo que más he podido trabajar durante el desarrollo del trabajo en equipo ha sido la capacidad o necesidad de escuchar activamente y de ponerme en situación del otro, de empatizar con las circunstancias personales de cada uno. Usualmente estaba acostumbrada a trabajar en ambientes de equipo donde no había tanta predisposición al debate ni poníamos tanta atención al lenguaje" (testimonio de estudiante 3, asign Cooperación curso 2016-2017)

\subsubsection{Elementos negativos evidenciados}

Si bien la sensación general es que los mecanismos aplicados han permitido que el alumnado no solo lleve a la práctica el trabajo en equipo, sino que reflexione sobre él y desarrolle (de forma limitada) dimensiones más relacionadas con el enfoque de cuidados, también han aflorado valoraciones negativas durante el desempeño. En primer lugar, es unánime la opinión de que el tiempo dedicado es insuficiente (o excesivo, si se lo compara con el que se supone deben invertir respecto a las horas docentes y discentes previstas en el plan de estudios), y que es realmente difícil cuadrar agendas y abrir espacios de reflexión interna, más allá de realizar las tareas definidas para cada equipo. Este aprendizaje es de gran interés, no solo para modificar los métodos empleados por el profesorado, sino para que ellos mismos/as experimenten lo que les sucederá cuando desempeñen su actividad profesional, especialmente en contextos complejos y con elevada incertidumbre.

También se identificaron aspectos negativos de carácter actitudinal observados en algunas personas de los equipos: pasividad, responsabilidad diluida, personalidades dominantes, egocentrismo. Esto les permitió evidenciar que el trabajo en equipo depende en gran parte de lo que cada miembro sea como persona y proyecte al grupo y les animó a solicitar un segundo 
taller formativo. Sin duda, los espacios de reflexión provocados permitieron identificar estos elementos que consideraron no deseables desde los enfoques trabajados.

\subsection{Resultados relativos al sistema de implementación y evaluación definido}

\subsubsection{Adecuación del seguimiento}

En primer lugar, se aprecian diferencias en cuanto a si el trabajo en equipo se realizaba de manera presencial (cuatrimestre B) o no presencial (cuatrimestre A). Para el segundo caso, al no disponerse de espacio específico en aula para su realización, ello dificultó el seguimiento y favoreció que el trabajo fuera realizado de manera no constante por cada equipo. Aunque sí ha habido buen trabajo en equipo y el alumnado está en general muy contento y sorprendido por el clima logrado, la profesora no ha dispuesto de información completa del transcurso de las tareas. La profesora, así mismo, consideró que cada equipo debía ser autónomo como para solicitar reuniones de seguimiento, no provocadas por así decirlo, cosa que no ocurrió. Esto denota una no interiorización de que se estaba trabajando esta competencia.

En relación a la reunión de evaluación final, ésta fue muy importante, ya que salieron a la luz conflictos habidos en los equipos y se evidenció que, de forma autónoma (como se ha expuesto), ningún equipo de trabajo había dedicado tiempo expreso a dialogar sobre la marcha del trabajo en equipo. Al ser planteada en el cuatrimestre A, se identifica como posible causa la dificultad de atender gran cantidad de procesos nuevos para estudiantes que recién inician el título, con el añadido de la diferencia cultural al ser el alumnado de procedencias diversas; en ese sentido, lo importante para ellxs era lograr alcanzar los objetivos del trabajo, que velar por monitorizar la dinámica propiamente dicha del equipo.

\subsubsection{Flexibilidad en la evaluación}

Respecto al trabajo presencial llevado a cabo en la asignatura Gestión del Ciclo de Proyecto de Cooperación al Desarrollo (cuatrimestre B), de momento solo se ha podido realizar la evaluación intermedia pues la asignatura no ha finalizado. Como ya se expuso, fue el propio alumnado del máster quien consideró más interesante en el cuatrimestre $\mathrm{B}$ evaluar al equipo como conjunto, además de autoevaluarse. Esto se puede deber a que la evaluación en el cuatrimestre B conllevaba calificación para la nota o que bien no querían personalizar las opiniones, sino valorar el trabajo realizado por cada grupo, sin particularizar a cada miembro, y no romper así la buena sintonía de los grupos. Curiosamente la mayor parte del alumnado valoró mejor al equipo que así mismos. Esto confirma la buena sintonía y ambiente en los grupos.

Otro aprendizaje relacionado con la evaluación fue el hecho de que el profesor responsable de la asignatura había planificado al final de la clase 40 minutos para la evaluación: 20 minutos de aula para reflexionar por grupos (los mismos grupos que los realizados durante el trabajo en equipo de la asignatura) y otros 20 minutos para rellenar las hojas de

(c)) EY-NC-ND 2017, Universitat Politècnica de València 
autoevaluación y evaluación del equipo. Sin embargo, el alumnado, de manera dialógica y reflexiva, sugirió que preferían dedicar los últimos 20 minutos a una reflexión grupal de toda la clase para compartir las reflexiones de cada grupo y rellenar personalmente fuera del aula la autoevaluación y la coevaluación. Fruto de esta última reflexión grupal surgieron elementos muy interesantes relacionados con el enfoque de trabajo colaborativo que queremos destacar en esta comunicación. A modo de ejemplo, un grupo que había tenido ciertos problemas durante el trabajo en equipo, porque una persona siempre mostraba una opinión diferente al resto, comentó lo siguiente:

"Gracias al enfoque que se nos ha inculcado desde el máster hemos convertido esa debilidad en una fortaleza, aprendiendo de las diferencias de opinión como grupo y creando debates más constructivos" (testimonio de equipo de trabajo, curso 2016-2017)

\subsection{Resultados globales}

Como fue expuesto anteriormente, trasladar a una competencia transversal como es el trabajo en equipo elementos que se alejan de un modelo que podríamos calificar como eficientista, supone reinterpretar los marcos conceptuales y de trabajo en el aula, deconstruyendo ideas y experiencias previas y animando al alumnado a que participe activamente y a que realice un trabajo reflexivo en paralelo a su estudio habitual. Para quienes suscriben la presente comunicación, está siendo alentador ver cómo el alumnado potencia sus capacidades personales (lo cual se traslada a las colectivas), si la mirada no solo considera elementos esenciales del trabajo en equipo (como la responsabilidad, la cooperación o la gestión), sino que incorpora la educación emocional y el enfoque de cuidados: comprensión de la situación de la otra persona, empatía o compañerismo, entre otros.

Como valoración global, se evidencia como esencial continuar con la aplicación del enfoque y herramientas participativas en el máster, lo que permite un co-aprendizaje y potenciar su autonomía y responsabilidad; pero también es necesario seguir considerando que el trabajo en equipo es un espacio básico para generar cultura de la participación, concepto que también es impartido en el MCAD como dimensión básica del desarrollo humano sostenible. Todo ello desde un enfoque más político, ligado al empoderamiento y al aumento de la libertad para ser capaces de contribuir a la transformación de la realidad.

\section{Conclusiones}

Si bien la adopción de la competencia transversal trabajo en equipo y liderazgo en cualquier titulación universitaria es ya un hecho y una opción ineludible, consideramos imprescindible una reflexión sobre el enfoque desde el cual esta competencia es interpretada, si queremos contribuir a la formación de profesionales que sean capaces de llevar a la práctica valores y actitudes más cercanos al desarrollo humano sostenible. Tal es el caso de la solidaridad frente a la competitividad exacerbada o bien la empatía, la sensibilidad, la comprensión y el cuidado del otro/a frente a la desafección por las situaciones de las otras personas que nos rodean, la apatía o la exclusión de las emociones en los espacios profesionales. 
Adoptar el enfoque de la participación y de cuidados para reinterpretar el trabajo en equipo en las aulas universitarias es una de las vías que consideramos pueden hacernos transitar hacia una cultura menos gerencialista y eficientista. A pesar de disponer de evidencias limitadas que no nos permiten hacer afirmaciones totalmente contrastadas, sí estamos constatando con la adopción de ambos enfoques en esta competencia transversal en el máster, que el alumnado demanda una nueva visión del trabajo en equipo y valora positivamente las mejoras desarrolladas. Visión que tome en consideración su ser como sujetos con capacidad de participar, y que no pueden eludir (aunque sí tratar de controlar) sus estados emocionales y personales cuando afrontan una tarea en equipo. Esta necesidad de reorientar el trabajo en equipo requiere, a su vez, de redefinir las asignaturas del plan de estudios, puesto que no se trata solamente de una cuestión de mejora de los métodos de evaluación, sino de llegar realmente a incorporar de forma transversal y coordinada esta competencia. En el caso que nos ocupa, la existencia de un equipo de innovación docente en el seno del máster, así como los propios planteamientos del mismo (visión crítica, participación, etc.) favorecen sin duda que ello pueda lograrse. De igual modo, la colaboración universidad-tercer sector, en este caso UPV-ONG de Desarrollo, permite trasladar la realidad al aula y construir de forma conjunta ese espacio educativo con vocación transformadora.

Finalmente, se evidencian diversas necesidades a abordar, como pueden ser: sistematizar las experiencias como práctica institucionalizada; profundizar en los enfoques adoptados y su traslado a la competencia estudiada; mantener la colaboración, y con carácter más estratégico, con agentes y entidades externas; lograr que la universidad comprenda la dotación de recursos que se precisa para abordar adecuadamente el trabajo en equipo como competencia transversal.

\section{Referencias}

ÁBALOS, A. et al. (2015). Rúbrica UPV CT-06. trabajo en equipo y liderazgo. Accesible en $<$ http://poliformat.upv.es $>$ [Consulta: marzo de 2016]

BELDA, S., BONI, A., PERIS, J. y TEROL, L. (2012). "Capacity development for emancipator social change. Reimaging university learning and teaching for critical development practitioners", en Journal of International Development, vol. 24, p. 571-584.

BELDA, S. PERIS, J., y CUESTA, I. (2016). "Participation for transformative learning in development management: The case of a Master in international development projects in the Universitat Politècnica de València (Spain)". Comunicación en el 2nd International Conference on Higher Education Advances, HEAd'16, 21-23 June 2016, València, Spain.

BONI-ARISTIZABAL, A., CALABUIG-TORMO, C. y PELLICER SIFRES, V. (2017). "Transforming the system from within: the experience of the Development Cooperation", en Walker, M. y Wilson-Strydom, M. (Eds.) Socially Just Pedagogies, Capabilities and Quality in Higher Education, London: Palgtrave MacMillan.

CARRASCO, C., BORDERÍAS, C., y TORNS, T. (Eds) (2011). El trabajo de los cuidados. Historia, teoría y políticas. Madrid: Los libros de la catarata.

CLARKE, P., y OSWALD, K. (2010). "Why reflect collectively on capacities for change?" en IDS Bulletin, issue 41, p. 1-12.

(cc) EY-NC-ND 2017, Universitat Politècnica de València 
DE BLAS, A. (2016). La revolución de los cuidados. Tácticas y estrategias. Madrid: InteRed.

EYBEN, R., GUIJT, I., ROCHE, C. y C. SHUTT (Eds.) (2015). The Politics of Evidence and Results in International Development: Playing the game to change the rules?. Rugby: Practical Action Publishing.

GULRAJANI, N. (2010). "New vistas for development management: examining radical-reformist possibilities and potential" en Public Administration and Development, vol. 30, p. 136-148.

HERRERO, Y. (2013). "Miradas ecofeministas para transitar a un mundo justo y sostenible" en Revista de Economía Crítica, ${ }^{\circ} 16$, segundo semestre 2013, p. 278-307.

JOHNSON, H. y THOMAS, A. (2007). "Individual learning and building organizational capacity for development" en Public Administration and Development, vol. 27, p. 39-48.

MCCOURT, W., y GULRAJANI, N. (2010). “The future of development management: Introduction to the Special Issue" en Public Administration and Development, vol.30, p. 81-90.

MOSSE, D. (2005). Cultivating development: An ethnography of aid policy and practice. London: Pluto Press.

ORTIZ ARAGON, A. (2010). "A case for surfacing theories of change for purposeful organizational capacity development" en IDS Bulletin, vol.41, p. 36-46.

PELLICER SIFRES, V.; CALABUIG TORMO, C. (2015). "El potencial de los espacios educativos informales en la universidad para una práctica crítica del desarrollo", en $4^{\circ}$ Congreso Internacional Multidisciplinar de Investigación Educativa (CIMIE 2015). Valencia, España.

PERIS, J., BELDA, S., y CUESTA, I. (2013). "Educating development professionals for reflective and transformative agency". En Boni A. \& Walker M. (Eds.). Human Development and Capabilities: Reimagining the University of the Twenty-first Century, London and New York: Routledge.

SOAL, S. (2010). "The more things change, the more they stay the same?", en IDS Bulletin, vol.41, p. $128-137$.

PEARSON, J. (2010). "Pushing at a half-open door" en IDS Bulletin, vol. 41, p. 118-127.

PETTIT, J. (2006). "Power and pedagogy: Learning for reflective development practice" en IDS Bulletin, vol.37, p. 69-78. 